\title{
Effect of high-fat diet-induced obesity on the Akt/FoxO/Smad signaling pathway and the follicular development of the mouse ovary
}

\author{
YANQING WU, ZHENGHONG ZHANG, XINGHUI LIAO, LINGBIN QI, YIPING LIU and ZHENGCHAO WANG
}

\author{
Provincial Key Laboratory for Developmental Biology and Neurosciences, \\ College of Life Sciences, Fujian Normal University, Fuzhou, Fujian 350007, P.R. China
}

Received July 16, 2015; Accepted August 10, 2016

DOI: $10.3892 / \mathrm{mmr} .2016 .5671$

\begin{abstract}
Obesity has a negative effect on ovarian functions, which is reported to increase the risk of infertility. The mechanism underlying obesity-induced infertility is not yet clear. The present study established a high-fat diet (HFD)-induced obesity mouse model to elucidate the mechanisms underlying the effect of HFD-induced obesity on follicular development in the mouse ovary. The 4-week-old female mice were fed with HFD or normal control (NC) diet for 15 or 20 weeks. Body mass index was used to demonstrate that the mice were obese following HFD treatment. The follicular development of the ovaries from the HFD group mice was retarded in a time-dependent manner, as demonstrated by morphological and histological examination of the ovaries. Further investigation via western blot analysis demonstrated that the activity of the transcription factor, forkhead box O3a (FoxO3a), was increased by HFD through downregulated FoxO3a phosphorylation, which may contribute to the inhibited development of ovarian follicles. To determine the regulatory mechanism of FoxO3 on the follicular development, the expression levels of FoxO3a target protein, Smad1/5/8, were also determined and there was significant decrease in phosphorylated Smad1/5/8 in the ovaries from the HFD group compared with the NC group, indicating that FoxO3a/Smad1/5/8 may be important in the regulation of follicular development. The expression levels of the upstream regulator of FoxO3a, Akt, were also examined and it was demonstrated that Akt phosphorylation was significantly reduced in the HFD group compared with the $\mathrm{NC}$ group, indicating that $\mathrm{Akt} / \mathrm{FoxO} 3$ a may be also involved in follicular development. Together, the experiments
\end{abstract}

Correspondence to: Dr Zhengchao Wang, Provincial Key Laboratory for Developmental Biology and Neurosciences, College of Life Sciences, Fujian Normal University, 8 Shangsan Road, Fuzhou, Fujian 350007, P.R. China

E-mail: zcwang@fjnu.edu.cn

Key words: high-fat diet, obesity, Akt, FoxO3a, Smad1/5/8, follicular development demonstrated that HFD-induced obesity affected the activity of the Akt/FoxO3a/Smad1/5/8 signaling pathway in a time-dependent manner during the follicular development of the mouse ovary, leading to abnormal follicular development. These findings may provide part of a theoretical basis for the clinical prevention and treatment of obesity-associated female infertility.

\section{Introduction}

The increasing prevalence of obesity has become a major worldwide issue. Obesity is associated with high rates of type 2 diabetes mellitus, cardiovascular disease and infertility $(1,2)$. The Nurses' Health study reported that a high body mass index (BMI) value is associated with a high risk of infertility in women (3). Furthermore, obesity is an important factor involved in polycystic ovary syndrome, a condition associated with anovulatory infertility (1,4-6). However, the molecular mechanism of the effect of obesity on follicular development remains unclear.

In the mammalian ovary, follicular development is regulated by the hypothalamus-pituitary-ovary axis and various other signaling pathways (7). As a vital transcription factor, forkhead box O3a (FoxO3a) negatively regulates primordial follicle activation and early follicular development $(8,9)$. Previous investigations have indicated that FoxO3a is highly expressed and localized to the nucleus during the dormancy of primordial follicles, then is phosphorylated and exported to the cytoplasm during follicular activation in mice (10). However, the activity of FoxO3a is suppressed in later-growing follicles, indicating that the downregulation of FoxO3a in oocytes may be a prerequisite for the initiation of oocyte growth during follicular activation $(11,12)$.

It is well-established that the bidirectional communication between oocytes and granulose cells (GCs) is also important for normal follicular development. Bone morphogenetic protein 15 (BMP15) and the transforming growth factor- $\beta$ family members, are fundamental for the paracrine signaling between oocytes and GCs that controls follicular development and cumulus expansion (13-18). GCs are one of the target cells of BMP15 $(19,20)$. BMPs bind to their cell membrane receptor and exert their effects through receptor-mediated 
activation of Smad transcription factors (21). Previous studies have demonstrated that BMP15 regulates Smad1/5/8 activity and regulates ovarian follicular development (22-24). Additionally, Liu et al $(11,25)$ reported that constitutively active FoxO3a expressed in oocytes stimulated downregulation of BMP15 expression and suppressed Smad pathway activation. Furthermore, the BMP15 gene promoter contains FoxO3a binding sites $(11,25)$. These previous studies indicated that the regulation of FoxO3a during follicular development may be mediated via the BMP15/Smad1/5/8 signaling pathway.

Previous studies have systematically reported that maintaining optimal activity of Akt serine/threonine kinase 1 (Akt) signaling is essential for normal ovarian function, which regulates the balance between survival and activation of primordial follicles, and also is important for proliferation and differentiation of GCs (26-28). FoxO3a is also a substrate of Akt, thus, the present study also examined the expression of Akt ovaries. The present study was designed to verify whether Akt/FoxO3a/Smad1/5/8 signaling is the intracellular mechanism underlying the effect of high-fat diet-induced obesity on the follicular development, to demonstrate the importance of understanding the effect of abnormal metabolite process in ovarian function and potentially elucidating novel interventions for obese pregnant mothers.

\section{Materials and methods}

Animals. Four-week old female C57BL/6J mice (weighing $\geq 35 \mathrm{~g}$ ) were purchased from Wushi Experimental Animal Supply (Fuzhou, China). The animals were maintained under a 14-h light/10-h dark condition, at $25^{\circ} \mathrm{C}$. The experimental protocol was approved in accordance with the Guide for the Care and Use of Laboratory Animals prepared by the Institutional Animal Care and Use Committee (Fujian Normal University, Fuzhou, China).

Experimental design. The mice $(\mathrm{n}=60)$ were divided into two groups (n=30/group) and fed either a high-fat diet (HFD; Research Diets, Inc., New Brunswick, NJ, USA) or a normal control diet (Research Diets, Inc.) for 15 or 20 weeks. HFD contained $20 \%$ protein, $20 \%$ carbohydrate and $60 \%$ fat. The normal control (NC) diet contained $20 \%$ protein, $70 \%$ carbohydrate and $10 \%$ fat. Body weight was recorded weekly. Following feeding with the different diets for 15 and 20 weeks, the BMI was determined. BMI was calculated using the following formula: body weight $/$ height ${ }^{2},\left(\mathrm{~kg} / \mathrm{m}^{2}\right)$. Following feeding with the different diets for 15 or 20 weeks, the mice were sacrificed with $20 \% \mathrm{CO}_{2}$, followed by cervical dislocation, and the ovaries were dissected from the mice. One ovary was examined morphology under a Leica MZ16F stereomicroscope (Leica Microsystems, Inc., Buffalo Grove, IL, USA) or via hematoxylin and eosin (H\&E) staining, and the other ovary was snap-frozen and used for immunoblotting.

$H \& E$ staining. Ovaries were collected from mice fed with the different diets for 15 or 20 weeks, and then fixed with $4 \%$ paraformaldehyde in phosphate-buffered saline. Ovaries were dehydrated in alcohol and embedded with paraffin. Sections $(10 \mu \mathrm{m})$ were stained with $\mathrm{H} \& \mathrm{E}$ and observed under a light microscope.

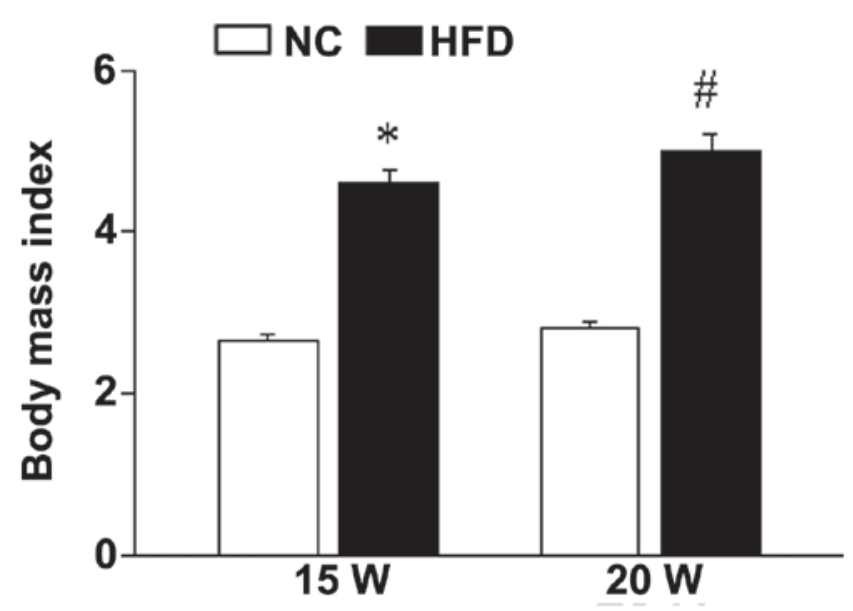

Figure 1. Animal model of HFD-induced obesity. Body mass index of mice in $\mathrm{NC}(15 \mathrm{~W}, \mathrm{n}=6 ; 20 \mathrm{~W}, \mathrm{n}=7)$ and HFD $(15 \mathrm{~W}, \mathrm{n}=6 ; 20 \mathrm{~W}, \mathrm{n}=8)$ groups following feeding with NC or HFD for 15 and 20 weeks ( $\mathrm{P}<0.05$ vs. $15 \mathrm{~W}$ group; ${ }^{\#} \mathrm{P}<0.05$ vs. $20 \mathrm{~W}$ NC groups). Data are presented as the mean \pm standard deviation. NC, normal control group; HFD, high-fat diet group; W, weeks.

Immunoblotting. Immunoblotting was performed as previously described (2,29-34). Briefly, ovaries from different experimental groups were sonicated in lysis buffer containing a protease inhibitor cocktail (Sigma-Aldrich, St. Louis, MO, USA). The proteins were quantified using DC protein assay kit (Bio-Rad Laboratories, Inc., Hercules, CA, USA). Equal amounts of protein $(30-50 \mu \mathrm{g})$ and the Precision Plus Protein Standards (Bio-Rad Laboratories, Inc.) were resolved by sodium dodecyl sulfate-polyacrylamide gel electrophoresis and transferred onto polyvinylidene difluoride membranes. Membranes were blocked in $5 \%$ nonfat milk for $45 \mathrm{~min}$ at $25^{\circ} \mathrm{C}$ and then incubated for $18 \mathrm{~h}$ at $4^{\circ} \mathrm{C}$ with the primary antibodies at 1:1,000 in 5\% non-fat milk, including Akt (cat. no. 8272), phosphorylated (p)-Akt (cat. no. 4058), FoxO3a (cat. no. 9467), p-FoxO3a (cat. no. 9464s), Smad1/5/8 (cat. no. 9517P) and p-Smad1/5/8 (cat. no. 9511) (all from Cell Signaling Technology, Inc., Danvers, MA, USA). To determine whether equivalent amounts of protein were loaded among all samples, membranes were stripped and incubated with a mouse antibody against $\beta$-actin (Abcam, Cambridge, UK) to generate a signal for use as a loading control. Following primary antibody incubation, the membranes were incubated with goat anti-rabbit or goat anti-mouse secondary antibodies (1:10,000; Jackson ImmunoResearch Laboratories, Inc., West Grove, PA, USA) at $25^{\circ} \mathrm{C}$ for $4 \mathrm{~h}$. Using the Super Signal West Femto Maximum Sensitivity Substrate kit (Thermo Fisher Scientific, Inc., Waltham, MA, USA), chemiluminescence emitted from the bands was directly captured using a UVP Bioimage EC3 system (UVP, Inc., Upland, CA, USA). Densitometric analysis of chemiluminescence signals was performed using VisionWorks LS software (version, EC3 600; UVP, Inc.). The quantitative analysis of protein levels was calculated as the densitometric value of phosphorylated protein level/the densitometric value of total protein. All experiments were repeated in triplicate with the use of independently prepared tissue lysates.

Statistical analysis. Data are presented as the mean \pm standard error. Experiments were repeated at least three times, and ovary samples for each replicate were from different female mice. 

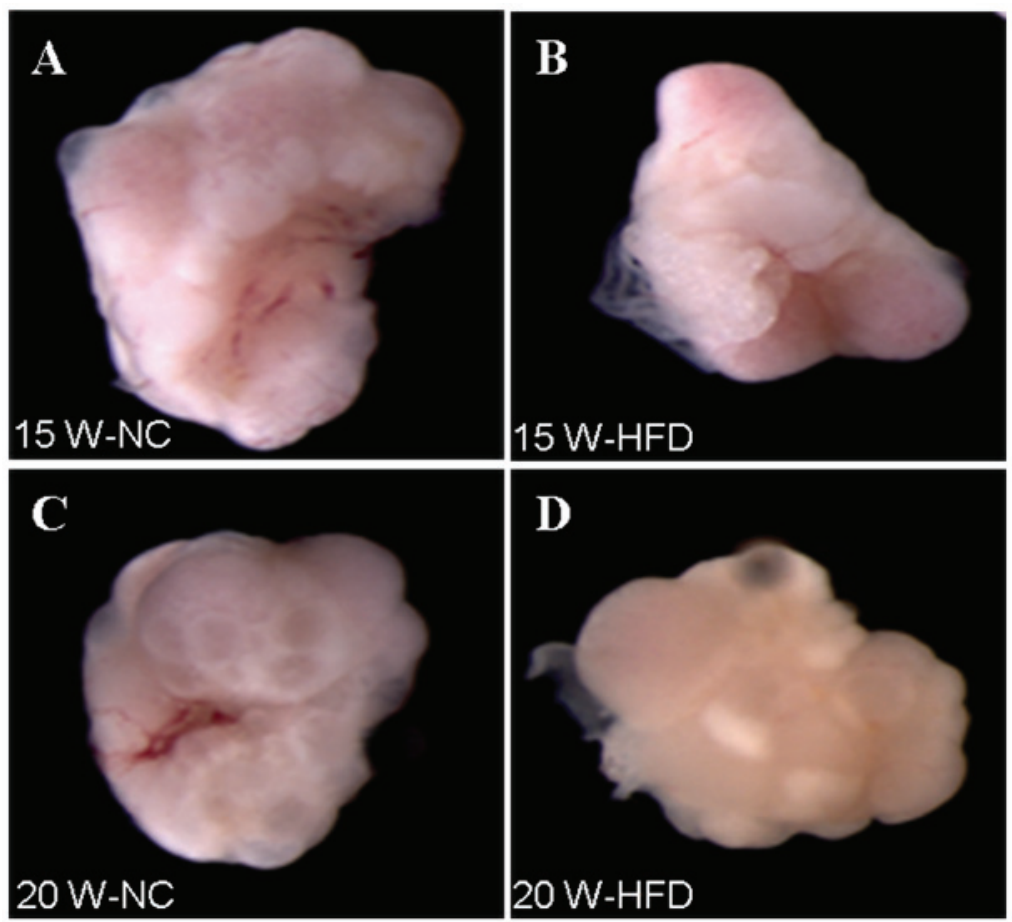

Figure 2. HFD-induced obesity results in abnormal ovarian morphology. Morphological appearance of ovaries from mice in (A) NC group and (B) HFD group following feeding with the different diets for 15 weeks; B is the morphological appearance of ovaries from mice in HFD group after fed with different diet for 15 weeks. Morphological appearance of ovaries from mice in (C) NC group and (D) HFD group following feeding with the different diets for 20 weeks. W, weeks; NC, normal control group; HFD, high-fat diet group.

Statistical differences were determined by one-way analysis of variance with Tukey's test used to determine the significance of the results using Sigma Stat 3.5 software (Systat Software, Inc., San Jose, CA, USA). P $<0.05$ was considered to indicate a statistically significant difference.

\section{Results}

Animal model of HFD-induced obesity. The mice received HFD or NC diet for 15 or 20 weeks. After 15/20-week feeding, the BMI of the mice in the HFD group was significantly increased compared with the NC group (15 weeks, $\mathrm{P}<0.001 ; 20$ weeks, $\mathrm{P}<0.001$ ), indicating that the obese mouse model has been successfully established (Fig. 1).

HFD-induced obesity causes abnormal ovarian morphology. HFD-induced obesity had a negative effect on ovarian function. The ovarian morphology in the HFD group (15 weeks) did not exhibit obviously abnormal ovarian morphology compared with the NC group (Fig. 2A and B). However, when administered with HFD for 20 weeks, an abnormal morphological effect of obesity on ovarian development was observed (Fig. 2C and D). H\&E staining was performed to observe the histology of the ovaries. The majority of the follicles in the ovaries from the $\mathrm{NC}$ group had developed to the later follicles stages or the corpora lutea stage (Fig. 3). By contrast, the mouse ovaries in the HFD group exhibited hyaline appearance, were avascular and had large numbers of cystic follicles stored under the tunica albuginea (Fig. 2D). Additionally, the majority of follicles in the ovaries from the HFD group (20 weeks) were retarded at an early stage or were atretic
(Fig. 3D). These findings suggested that HFD-induced obesity exerts a detrimental effect on follicular development.

$H F D$-induced obesity suppresses the phosphorylation level of FoxO3a in the ovary. Compared with the follicles in NC group, the majority of ovarian follicles were retarded at the early follicle stage in the HFD group (Fig. 3), indicating that the regulatory mechanisms underlying follicular development are disrupted. FoxO3a is a major regulator of primordial follicle activation and early follicular development. To determine whether FoxO3a is involved in the effect of HFD-induced obesity on follicular development, the expression level of p-FoxO3a in the ovaries was examined. The protein level of p-FoxO3a was significantly reduced in the ovaries from mice in the HFD group compared with the NC group at 15 weeks $(\mathrm{P}<0.001)$ and 20 weeks $(\mathrm{P}<0.001)($ Fig. 4). Additionally, the degree of reduction of p-FoxO3a in the ovaries from mice fed with HFD for 20 weeks was greater than in the ovaries from mice fed with HFD for 15 weeks; however, no difference in the total protein levels were observed compared with $\beta$-actin (15 weeks, $\mathrm{P}=0.002 ; 20$ weeks, $\mathrm{P}=0.629$; Fig. 4). These data suggest that HFD-induced obesity suppresses FoxO3a phosphorylation in a time-dependent manner, leading to increased FoxO3a activity and retarded follicular development.

HFD-induced obesity represses Smad signaling pathway activation in the ovary. BMP15 is specifically expressed in oocytes and is important for the regulation of cell growth, development and apoptosis $(19,20)$. FoxO3a can bind the BMP15 gene promoter to regulate its expression. The expression of $\mathrm{p}-\mathrm{Smad} 1 / 5 / 8$, the downstream effector of BMP15, was 

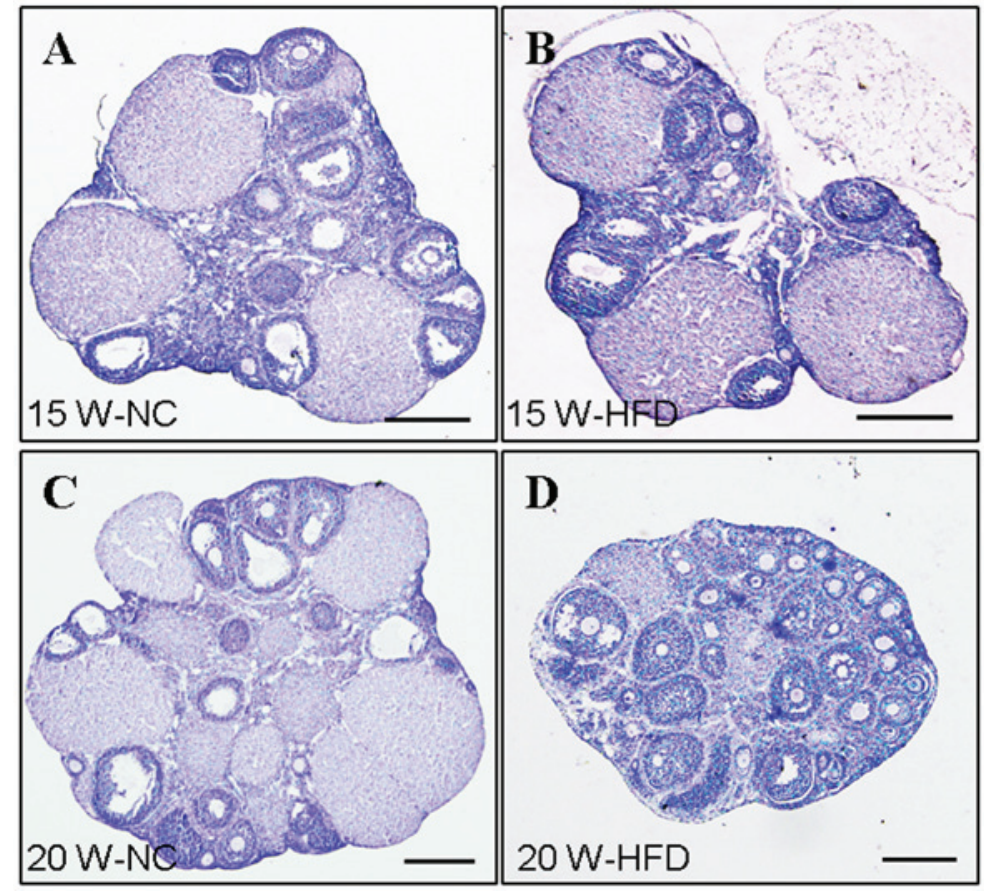

Figure 3. HFD-induced obesity resulted in abnormal ovarian histology. Representative images of ovarian histological structure from mice in (A) NC group and (B) HFD group following feeding with the different diets for 15 weeks. (C) Representative images of ovarian histological structure from mice in (C) NC group and (D) HFD-diet group following feeding with the different diets for 20 weeks. Scale bars=150 $\mu \mathrm{m}$. W, weeks; NC, normal control group; HFD, high-fat diet group.

A

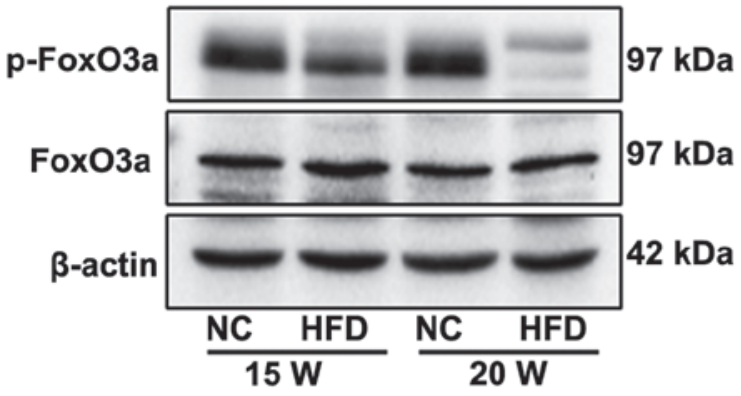

C

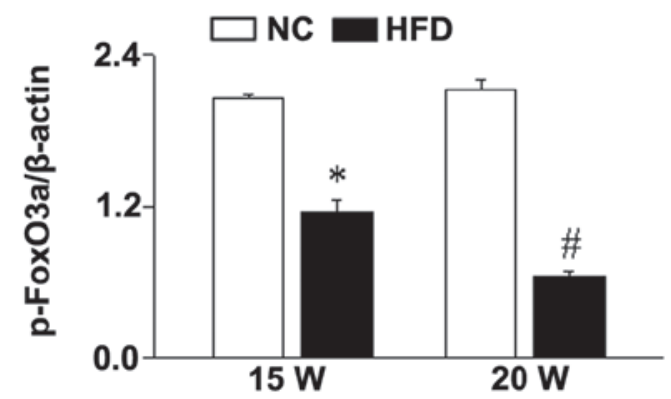

B

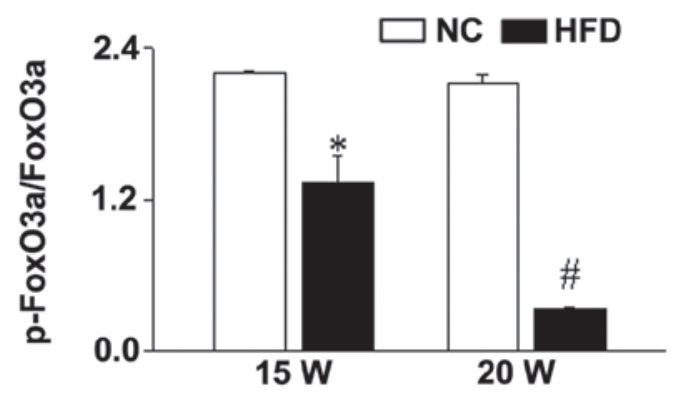

D

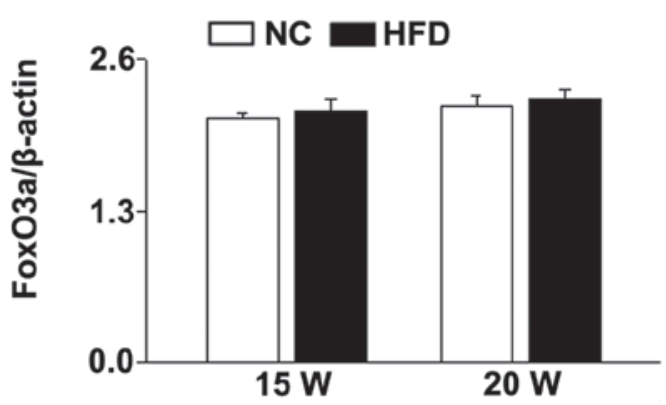

Figure 4. HFD-induced suppressed the phosphorylation level of FoxO3a in the ovary Protein levels of p-FoxO3a in the ovaries from mice in NC group and HFD group were measured following feeding with the different diets for 15 or 20 weeks. (A) Representative images of western blot analyses of the p-FoxO3a and total FoxO3a protein levels. (B) Intensities of p-FoxO3a blots normalized to FoxO3a. (C) Intensities of p-FoxO3a blots normalized to $\beta$-actin. (D) Intensities of FoxO3a normalized to $\beta$-actin. Data are presented as the mean \pm standard deviation ( $\mathrm{n}=3$; ${ }^{*} \mathrm{P}<0.05 \mathrm{vs}$. $15 \mathrm{~W}$ group; ${ }^{*} \mathrm{P}<0.05 \mathrm{vs}$. $20 \mathrm{~W}$ NC groups). p-FoxO3a, phosphorylated-forkhead box O3a; NC, normal control group; HFD, high-fat diet group; W, weeks.

significantly reduced in the ovaries from HFD mice compared with $\mathrm{NC}$ mice in a time-dependent manner (15 weeks, $\mathrm{P}<0.001$; 20 weeks; $\mathrm{P}<0.001$; Fig. 5). Thus, these data indicate that FoxO3a/Smad1/5/8 signaling may be involved in the effect of HFD-induced obesity on follicular development.
HFD-induced obesity downregulates Akt signaling in the ovary. Akt, an upstream regulator of FoxO3a, is essential for the adverse metabolic effects of insulin, which is the major regulatory mechanism involved in follicular development (25). Thus, the effect of HFD-induced obesity on Akt signaling 


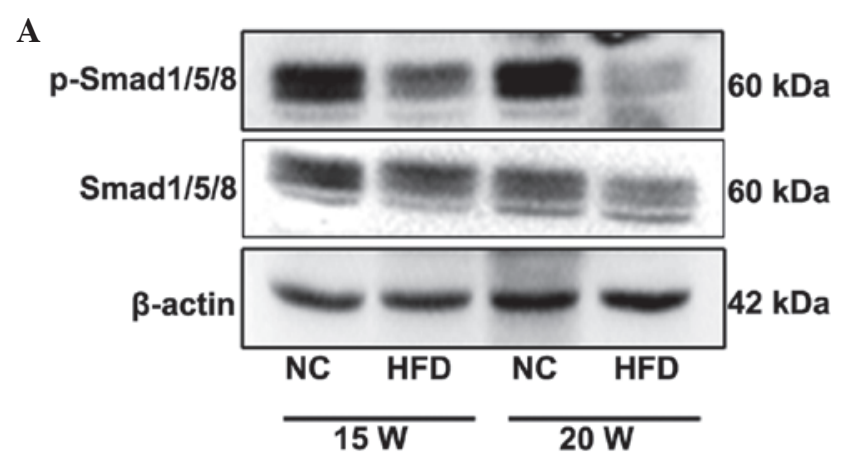

B

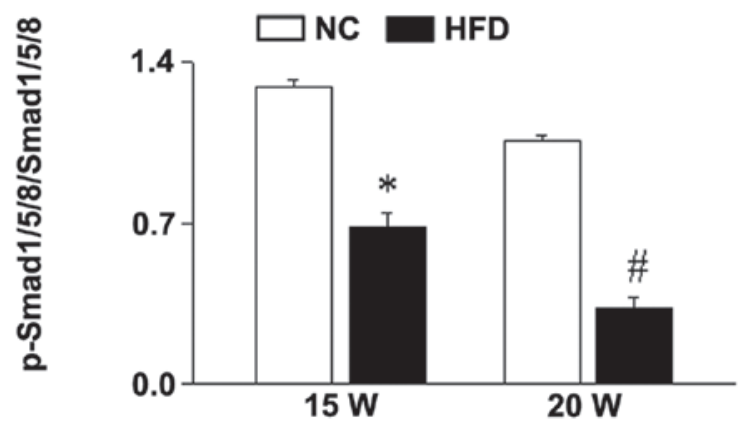

Figure 5. HFD-induced obesity represses Smad signaling activation in the ovary Protein levels of p-Smad1/5/8 in the mouse ovaries from NC group and HFD group following feeding with the different diets for 15 or 20 weeks. (A) Representative images of western blot analyses of $\mathrm{p}$-Smad1/5/8 and Smad1/5/8 protein levels. (B) Intensities of $\mathrm{p}$-Smad1/5/8 blots normalized to Smad1/5/8. Data are presented as the mean \pm standard deviation $(n=3$; ${ }^{*} \mathrm{P}<0.05$ vs. $15 \mathrm{~W}$ group; ${ }^{*} \mathrm{P}<0.05$ vs. $20 \mathrm{~W} \mathrm{NC}$ groups). $\mathrm{p}$-, phosphorylated; NC, normal control group; HFD, high-fat diet group; W, weeks.

during the follicular development was investigated. The level of p-Akt was significantly reduced in the ovaries from HFD group mice compared with the $\mathrm{NC}$ group (15 weeks, $\mathrm{P}=0.038$; 20 weeks, $\mathrm{P}<0.001$; Fig. 6 ). In accordance with the expression level of p-FoxO3a, the reduction of p-Akt expression in the ovaries from the mice fed with HFD for 20 weeks was increased compared with the mice fed with HFD for 15 weeks (Fig. 6). These data suggested that HFD-induced obesity may cause abnormal follicular development via impairing Akt/FoxO3a signaling in a time-dependent manner during follicular development.

\section{Discussion}

The results of the present study demonstrated that HFD-induced obesity observably inhibited follicular development in the mouse ovary in a time-dependent manner. Furthermore, HFD increased FoxO3a activity in the mouse ovaries, as indicated by decreased $\mathrm{p}-\mathrm{FoxO} \mathrm{O}$ a levels. These results together indicated that FoxO3a may be an important regulatory factor involved in the ovarian follicular development of HFD-fed mice.

Obesity is currently the major factor that causes type 2 diabetes, cardiac malformation and infertility (1). With the increasing epidemic of obesity, a useful mouse model of obesity is essential for studying the mechanisms that underlie the negative effects of obesity negatively on the function of the ovary. The current study established an obese mouse model
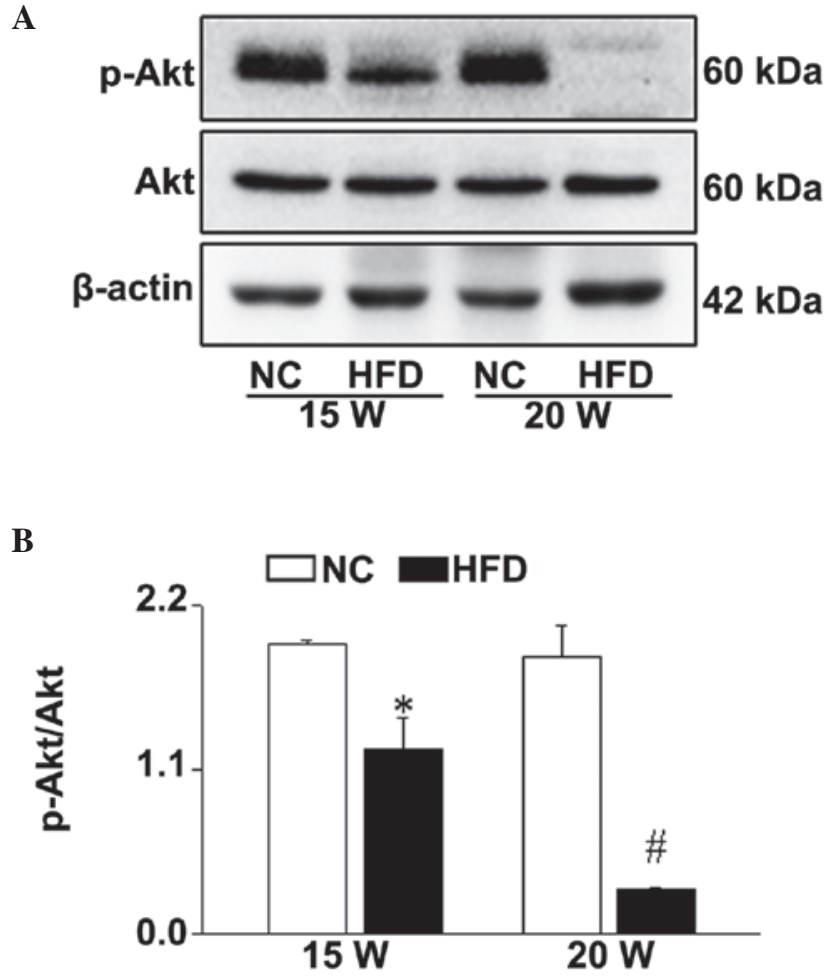

Figure 6. HFD-induced obesity downregulates Akt signaling in the ovary Protein levels of p-Akt in the mouse ovaries from NC group and HFD group were determined following feeding with the different diets for 15 or 20 weeks. (A) Representative of western blot analyses depicting the p-Akt and Akt protein levels. (B) Intensities of p-Akt blots normalized to Akt. Data are presented as the mean \pm standard deviation $\left(n=3 ;{ }^{*} \mathrm{P}<0.05\right.$ vs. $15 \mathrm{~W}$ group; ${ }^{\#} \mathrm{P}<0.05$ vs. $20 \mathrm{~W}$ NC groups). p-Akt, phosphorylated Akt serine/threonine kinase 1; NC, normal control group; HFD, high-fat diet group; W, weeks.

using C57BL/6J female mice fed with HFD for 15 or 20 weeks and focused on the molecular mechanism underlying the effect of obesity on follicular development. The present results demonstrated that HFD induced-obesity has deleterious effect on the ovarian development in a time-dependent manner. The follicular development in mice fed with HFD for 20 weeks was retarded at the early stage or were completely atretic. Thus, it is important to investigate the mechanisms underlying these processes.

In the mammalian ovary, follicular development is regulated by the hypothalamus-pituitary-ovary axis, and various signaling pathways (7). Ovarian follicles develop from primordial follicles to primary follicles, secondary follicles and antral follicles, which become a mature follicle with a single oocyte enclosed by several layers of somatic granulose cells (10). A substantial process is involved in the regulation of follicular development. In the ovary, primordial follicles are the source of all developing follicles, which have three potential developmental fates: i) To remain quiescent; ii) to be activated into the growing follicle pool; or iii) to undergo death directly from the dormant state (35-37). Consequently, the balance among these three fates of the primordial follicle determines the length of female reproductive life $(27,37,38)$. Additionally, the bidirectional communication between oocytes and GCs is also important for normal follicular development.

As a vital transcription factor, FoxO3a negatively regulates primordial follicle activation and early follicular 
development $(8,9)$. It has been previously demonstrated that phosphorylation by phosphoinositide 3 kinase (PI3K)/Akt controls a shuttling system that modulates FoxO3a nucleocytoplasmic translocation and, thus, its activity. Under normal conditions, FoxO3a is located in the nucleus and activates the expression of genes associated with cell death/apoptosis (39). Following stimulation, Akt phosphorylates FoxO3a, leading to the association of FoxO3a with 14-3-3 proteins, retention of FoxO3a in the cytoplasm and inhibition of FoxO3a-dependent transcriptional activity $(25,39)$. Regarding follicular development, FoxO3a may activate the expression of certain genes that maintain follicle quiescence. A number of elegant genetic mouse experiments have provided insight into the role of FoxO3a in oocyte quiescence. FoxO3a $\mathrm{a}^{-/-}$mice exhibited global activation of primordial follicles and age-dependent infertility $(8,40)$. Oocyte-specific ablation of phosphatase and tensin homolog (a negative regulator of PI3Ks) led to PI3K-induced Akt activation, and thus, phosphorylated FoxO3a, suppressing its activity, and consequently triggering a phenotype in oocytes equivalent to that in mice lacking FoxO3a $(41,42)$. The reduced phosphorylation of FoxO3a observed in the present study would maintain FoxO3a localization in the nuclei, which prevents the activation of follicle development.

Previous studies have also demonstrated that constitutively active FoxO3a suppressed Smad pathway activation via downregulation of BMP15 expression in the ovaries from ZP3-FoxO3a Tg mice (11). BMP15 is a growth factor specifically secreted from oocytes, which is important for promoting follicular development (13-18). GC is one of the target cells of BMP15 (19,20). Otsuka et al (43) demonstrated that BMP15 controls the expression of plasma protein-A in granulose cells coordinating with follicle stimulating hormone, guaranteeing the development of the advantage follicle. Additionally, BMP15 and growth differentiation factor-9 are required for cholesterol synthesis and metabolism in cumulus cells, adversely affecting the development of cumulus oocyte complexes (22). These findings indicated that FoxO3a/BMP15 signaling may be an important mechanism that regulates follicular development. Notably, previous studies have demonstrated that BMP15 regulates Smad1/5/8 activity and its regulatory effect on ovarian follicular development (22-24). Thus, the present study investigated Smad1/5/8 activity and demonstrated that HFD significantly reduced the level of p-Smad1/5/8. These studies suggest that HFD-induced obesity may impair FoxO3a/Smad1/5/8 signaling and lead to abnormal follicular development. The present data and previous results reinforce the importance of bidirectional communication between oocytes and somatic cells during oocyte and follicle development, and also reveal that the signaling pathway and hypothalamus-pituitary-ovary axis synergistically regulate follicular development.

$\mathrm{PI} 3 \mathrm{~K} / \mathrm{Akt}$ signaling is one of the classic signaling pathways in mammalian cells. Following stimulation, PI3K is activated and then phosphorylates the third position hydroxyl group of the inositol ring of phosphatidylinositol, resulting in the production of second messengers of inositol lipid substances, including PIP2 and PIP3 (44,45). Akt, also known as protein kinase $\mathrm{B}$, is a direct downstream target of PI3K $(46,47)$. Upon binding to the pleckstrin homology domains in the N-terminus in the cell membrane with PIP3, Akt is phosphorylated and activated by the catalysis of 3-phosphoinositide-dependent kinases (48). Activation of Akt, in turn, activates downstream signaling molecules, including FoxO family transcription factors, glycogen synthase kinase 3, Bcl-2 family proteins and mammalian target rapamycin, to regulate physiological processes, including cell proliferation, differentiation and apoptosis (10).

Additionally, studies using a transgenic mouse model with constitutively active FoxO3a expressed in oocytes demonstrated that over-expression of FoxO3a impaired oocyte growth and folliculogenesis, leading to female infertility (11). John et al (12) reported that as the downstream of PI3K/Akt, hyperphosphorylation of FoxO3a induced over-activation of primordial follicules $(8,26,28)$. As previous studies have systematically reported that maintaining optimal activities of PI3K/Akt signaling pathway is essential for normal ovarian function, which not only regulates the balance between survival and activation of primordial follicles, but also is vital for the proliferation and differentiation of GCs (26-28). These studies suggested that regulation of the optimal expression level of FoxO3a is important spatially and temporally during follicular development. The data from the present study demonstrated that HFD-induced obesity impairs Akt signaling and induces over-activation of FoxO3a in a time-dependent manner, implying that Akt/FoxO3a signaling is a critical signaling pathway underlying HFD-induced obesity resulting in abnormal follicular development.

In summary, the current study revealed the molecular regulatory mechanisms underlying the effect of HFD-induced obesity on follicular development, highlighting the importance of Akt/FoxO3a/Smad1/5/8 signaling during regulation of follicular development. The results from this study may provide a theoretical basis for clinical treatment of infertility caused by obesity. As the regulation of biological processes is complicated, further investigation of the effect of HFD-induced obesity on the regulation of hormones and inflammatory factors during ovarian follicular development is required.

\section{Acknowledgements}

This work was supported by the National Natural Science Foundation of China (grant nos. 31101032 and 31271255), Program for New Century Excellent Talents in University of Ministry of Education of China (grant no. NCET-120614), Doctoral Foundation of the Ministry of Education in China (grant no. 20113503120002) and Fujian Provincial Science and Technology Projects of the Department of Education (grant no. JB14041).

\section{References}

1. Akamine EH, Marçal AC, Camporez JP, Hoshida MS, Caperuto LC, Bevilacqua E and Carvalho CR: Obesity induced by high-fat diet promotes insulin resistance in the ovary. J Endocrinol 206: 65-74, 2010 .

2. Wu Y, Wang F, Fu M, Wang C, Quon MJ and Yang P: Cellular stress, excessive apoptosis and the effect of metformin in a mouse model of type 2 diabetic embryopathy. Diabetes 64: 2526-2536, 2015.

3. Wamae CN: Advances in the diagnosis of human lymphatic filariases: A review. East Afr Med J 71: 171-182, 1994.

4. Metwally M, Li TC and Ledger WL: The impact of obesity on female reproductive function. Obes Rev 8: 515-523, 2007.

5. Legro RS: Evaluation and Treatment of Polycystic Ovary Syndrome. Practice 17: 82-85, 2000. 
6. Pasquali R and Casimirri F: The impact of obesity on hyperandrogenism and polycystic ovary syndrome in premenopausal women. Clin Endocrinol (Oxf) 39: 1-16, 1993.

7. Richards JS and Pangas SA: The ovary: Basic biology and clinical implications. Journal Clin Invest 120: 963-972, 2010

8. Castrillon DH, Miao L, Kollipara R, Horner JW and DePinho RA: Suppression of ovarian follicle activation in mice by the transcription factor Foxo3a. Science 301: 215-218, 2003.

9. Nteeba J, Ross JW, Perfield JW II and Keating AF: High fat diet induced obesity alters ovarian phosphatidylinositol-3 kinase signaling gene expression. Reprod Toxicol 42: 68-77, 2013.

10. Uhlenhaut $\mathrm{NH}$ and Treier M: Forkhead transcription factors in ovarian function. Reproduction 142: 489-495, 2011.

11. Liu L, Rajareddy S, Reddy P, Du C, Jagarlamudi K, Shen Y, Gunnarsson D, Selstam G, Boman K and Liu K: Infertility caused by retardation of follicular development in mice with oocyte-specific expression of Foxo3a. Development 134: 199-209, 2007.

12. John GB, Shirley LJ, Gallardo TD and Castrillon DH: Specificity of the requirement for Foxo3 in primordial follicle activation. Reproduction 133: 855-863, 2007.

13. Dong J, Albertini DF, Nishimori K, Kumar TR, Lu N and Matzuk MM: Growth differentiation factor-9 is required during early ovarian folliculogenesis. Nature 383: 531-535, 1996.

14. Elvin JA, Clark AT, Wang P, Wolfman NM and Matzuk MM: Paracrine actions of growth differentiation factor-9 in the mammalian ovary. Mol Endocrinol 13: 1035-1048, 1999.

15. Elvin JA, Yan C, Wang P, Nishimori K and Matzuk MM: Molecular characterization of the follicle defects in the growth differentiation factor 9-deficient ovary. Mol Endocrinol 13: 1018-1034, 1999.

16. Vitt UA, McGee EA, Hayashi $M$ and Hsueh AJ: In vivo treatment with GDF-9 stimulates primordial and primary follicle progression and theca cell marker CYP17 in ovaries of immature rats. Endocrinology 141: 3814-3820, 2000.

17. Vitt UA, Hayashi M, Klein C and Hsueh AJ: Growth differentiation factor-9 stimulates proliferation but suppresses the follicle-stimulating hormone-induced differentiation of cultured granulosa cells from small antral and preovulatory rat follicles. Biol Reprod 62: 370-377, 2000

18. Sánchez F and Smitz J: Molecular control of oogenesis. Biochim Biophys Acta 1822: 1896-1912, 2012.

19. Paulini F and Melo EO: The role of oocyte-secreted factors GDF9 and BMP15 in follicular development and oogenesis. Reprod Domest Anim 46: 354-361, 2011.

20. Gilchrist RB, Lane M and Thompson JG: Oocyte-secreted factors: Regulators of cumulus cell function and oocyte quality. Hum Reprod Update 14: 159-177, 2008.

21. McCulley DJ, Kang JO, Martin JF and Black BL: BMP4 is required in the anterior heart field and its derivatives for endocardial cushion remodeling, outflow tract septation, and semilunar valve development. Dev Dyn 237: 3200-3209, 2008.

22. Su YQ, Sugiura K, Wigglesworth K, O'Brien MJ, Affourtit JP, Pangas SA, Matzuk MM and Eppig JJ: Oocyte regulation of metabolic cooperativity between mouse cumulus cells and oocytes: BMP15 and GDF9 control cholesterol biosynthesis in cumulus cells. Development 135: 111-121, 2008.

23. Sugiura K, Su YQ, Diaz FJ, Pangas SA, Sharma S, Wigglesworth K, O'Brien MJ, Matzuk MM, Shimasaki S and Eppig JJ: Oocyte-derived BMP15 and FGFs cooperate to promote glycolysis in cumulus cells. Development 134: 2593-2603, 2007.

24. Wu YQ, Chen LY, Zhang ZH and Wang ZC: Effects of phosphatidylinositol-3 kinase/protein kinase b/bone morphogenetic protein-15 pathway on the follicular development in the mammalian ovary. Zhongguo Yi Xue Ke Xue Yuan Xue Bao 35 224-228, 2013 (In Chinese).

25. Liu H, Luo LL, Qian YS, Fu YC, Sui XX, Geng YJ, Huang DN, Gao ST and Zhang RL: FOXO3a is involved in the apoptosis of naked oocytes and oocytes of primordial follicles from neonata rat ovaries. Biochem Biophys Res Commun 381: 722-727, 2009.

26. Zheng W, Nagaraju G, Liu Z and Liu K: Functional roles of the phosphatidylinositol 3-kinases (PI3Ks) signaling in the mammalian ovary. Mol Cell Endocrinol 356: 24-30, 2012.

27. Adhikari D and Liu K: Molecular mechanisms underlying the activation of mammalian primordial follicles. Endocr Rev 30 : $438-464,2009$
28. Reddy KK, Lefkove B, Chen LB, Govindarajan B, Carracedo A, Velasco G, Carrillo CO, Bhandarkar SS, Owens MJ, Mechta-Grigoriou F and Arbiser JL: The antidepressant sertraline downregulates Akt and has activity against melanoma cells. Cell Melanoma Res 21: 451-456, 2008.

29. Yang P,Li X, Xu C, Eckert RL, Reece EA, Zielke HR and Wang F: Maternal hyperglycemia activates an ASK1-FoxO3a-caspase 8 pathway that leads to embryonic neural tube defects. Sci Signal 6 : ra74, 2013.

30. Wu Y, Wang F, Reece EA and Yang P: Curcumin ameliorates high glucose-induced neural tube defects by suppressing cellular stress and apoptosis. AM J Obstet Gynecol 212: 802 e801-e808, 2015.

31. Li X, Xu M, Pitzer AL, Xia M, Boini KM, Li PL and Zhang Y: Control of autophagy maturation by acid sphingomyelinase in mouse coronary arterial smooth muscle cells: Protective role in atherosclerosis. J Mol Med Berl 92: 473-485, 2014.

32. Wei YM, Li X, Xu M, Abais JM, Chen Y, Riebling CR, Boini KM, Li PL and Zhang Y: Enhancement of autophagy by simvastatin through inhibition of Rac1-mTOR signaling pathway in coronary arterial myocytes. Cell Physiol Biochem 31: 925-937, 2013.

33. Li X, Gulbins E and Zhang Y: Oxidative stress triggers Ca-dependent lysosome trafficking and activation of acid sphingomyelinase. Cell Physiol Biochem 30: 815-826, 2012.

34. Zhang Z, Pang X, Tang Z, Yin D and Wang Z: Overexpression of hypoxia-inducible factor prolyl hydoxylase-2 attenuates hypoxia-induced vascular endothelial growth factor expression in luteal cells. Mol Med Rep 12: 3809-3814, 2015.

35. McGee EA and Hsueh AJ: Initial and cyclic recruitment of ovarian follicles. Endocr Rev 21: 200-214, 2000.

36. Hansen KR, Knowlton NS, Thyer AC, Charleston JS, Soules MR and Klein NA: A new model of reproductive aging: The decline in ovarian non-growing follicle number from birth to menopause. Hum Reprod 23: 699-708, 2008.

37. Broekmans FJ, Knauff EA, te Velde ER, Macklon NS and Fauser BC: Female reproductive ageing: Current knowledge and future trends. Endocrinol Metab 18: 58-65, 2007.

38. Faddy MJ and Gosden RG: A model conforming the decline in follicle numbers to the age of menopause in women. Hum Reprod 11: 1484-1486, 1996.

39. Tzivion G, Dobson M and Ramakrishnan G: FoxO transcription factors; Regulation by AKT and 14-3-3 proteins. Biochim Biophys Acta 1813: 1938-1945, 2011.

40. Reddy P, Zheng W and Liu K: Mechanisms maintaining the dormancy and survival of mammalian primordial follicles. Endocrinol Metab 21: 96-103, 2010.

41. Reddy P, Liu L, Adhikari D, Jagarlamudi K, Rajareddy S, Shen Y, Du C, Tang W, Hämäläinen T, Peng SL, et al: Oocyte-specific deletion of Pten causes premature activation of the primordial follicle pool. Science 319: 611-613, 2008.

42. John GB, Gallardo TD, Shirley LJ and Castrillon DH: Foxo3 is a PI3K-dependent molecular switch controlling the initiation of oocyte growth. Dev Biol 321: 197-204, 2008.

43. Otsuka F, Yamamoto S, Erickson GF and Shimasaki S: Bone morphogenetic protein-15 inhibits follicle-stimulating hormone (FSH) action by suppressing FSH receptor expression. J Biol Chem 276: 11387-11392, 2001.

44. Wymann MP and Pirola L: Structure and function of phosphoinositide 3-kinases. Biochim Biophys Acta 1436: 127-150, 1998.

45. Whitman M, Downes CP, Keeler M, Keller T and Cantley L: Type I phosphatidylinositol kinase makes a novel inositol phospholipid, phosphatidylinositol-3-phosphate. Nature 332: 644-646, 1988

46. Burgering BM and Coffer PJ: Protein kinase B (c-Akt) in phosphatidylinositol-3-OH kinase signal transduction. Nature 376: 599-602, 1995.

47. Franke TF, Kaplan DR, Cantley LC and Toker A: Direct regulation of the Akt proto-oncogene product by phosphatidylinositol-3,4-bisphosphate. Science 275: 665-668, 1997.

48. Huang XL, Xu J, Zhang XH, Qiu BY, Peng L, Zhang M and Gan HT: PI3K/Akt signaling pathway is involved in the pathogenesis of ulcerative colitis. Inflamm Res 60: 727-734, 2011. 